\title{
X-ray spectrometry with Peltier-cooled large area avalanche photodiodes
}

\author{
L.M.P. Fernandes, J.A.M. Lopes, J.M.F. dos Santos *, C.A.N. Conde \\ Departamento de Física da Universidade de Coimbra, 3004-516 Coimbra, Portugal
}

\begin{abstract}
Performance characteristics of the response of a Peltier-cooled large-area avalanche photodiode are investigated. Detector gain, energy linearity, energy resolution and minimum detectable energy are studied at different operation temperatures. Detector energy resolution and lowest detectable X-ray energy present a strong improvement as the operation temperature is reduced from 25 to $15{ }^{\circ} \mathrm{C}$ and slower improvements are achieved for temperatures below $10{ }^{\circ} \mathrm{C}$.

(C) 2003 Elsevier B.V. All rights reserved.
\end{abstract}

PACS: $07.85 . \mathrm{Fv} ; 29.40 . \mathrm{Wk}$; $85.60 . \mathrm{Dw}$

Keywords: Avalanche photodiode; X-ray spectrometry; Peltier-cooling

\section{Introduction}

Large-area avalanche photodiodes (LAAPD) are compact, simple to operate monolithic devices made of silicon $\mathrm{p}-\mathrm{n}$ junctions. When a reverse high voltage is applied to the LAAPD the internal electric field increases with the depth, presenting a maximum around the $\mathrm{p}-\mathrm{n}$ junction. The electric field can reach values high enough to allow electron multiplication by impact ionisation [1-3]. An incident photon produces electron hole pairs and the resulting electrons are accelerated towards the $\mathrm{n}^{+}$-contact, undergoing avalanche multiplication due to the high electric field around the junction.

\footnotetext{
${ }^{*}$ Corresponding author. Tel.: +351-239-410667; fax: +351239-829158.

E-mail address: jmf@gian.fis.uc.pt (J.M.F. dos Santos).
}

LAAPDs have been used mainly as optical photosensors coupled to scintillators for X- and $\gamma$ ray detection in applications such as the electromagnetic calorimeter of the CMS detector in the LHC at CERN [4], nuclear physics [5] and PET instrumentation for medicine [6]. The application of LAAPDs to direct X-ray detection has also been investigated [7]. Since LAAPDs useful thickness for X-ray detection is only a few tens of micrometers, their detection efficiency decreases rapidly for X-ray energies above $5 \mathrm{keV}$, being about $45 \%, 25 \%, 8 \%$ and $4 \%$ for $8,10,15$ and 20 $\mathrm{keV}$, respectively.

The performance of LAAPDs for energy-dispersive X-ray fluorescence analysis was studied [8]. Energy resolutions similar to those of proportional counters were obtained and the minimum detectable X-ray energy (MDE) was in the 1-2 keV range. The LAAPD dark current affects the 
achieved detector energy resolution and limits the MDE. However, the LAAPD gain and dark current depend significantly on the temperature, a drawback for many practical applications. This dependence requires temperature control and stabilisation during measurements. Temperature stabilisation can be achieved by Peltier-cooling. LAAPDs with built-in Peltier-cooled devices are commercially available. On the other hand, the operation of LAAPDs at reduced temperatures may result in improved performance and lower X-ray energy detection limit: the LAAPD dark current can be reduced by about one order of magnitude when its temperature is reduced from room temperature to $0{ }^{\circ} \mathrm{C}$.

In this work we investigate the performance of a Peltier-cooled LAAPD for X-ray detection as a function of the temperature. The photodiode gain, energy resolution and MDE will be studied for different photodiode temperatures.

\section{Operational characteristics}

The Peltier-cooled LAAPD [9], with 16-mm diameter active area, was operated in a light-tight box to shield it from ambient light. The LAAPD signals were fed through a low-noise charge preamplifier (Canberra 2004, with a sensitivity of 45 $\mathrm{mV} / \mathrm{MeV}$ ) to a spectroscopy amplifier (Tennelec TC 243, with gains of 32-256 and 500 ns shaping time) and were pulse-height analysed by a 1024channel multi-channel analyser (Nucleus PCA II).

For pulse amplitude and energy resolution measurements, the X-ray pulse-height distributions were fitted to a Gaussian function superimposed on a linear background, from which the centroid and the full-width at half-maximum were determined. The electronic noise tail at low energies limits the MDE, which we define as the energy limit where the electronic-noise tail raises above the background level.

The operational characteristics of the LAAPD at different temperatures were evaluated with 5.9$\mathrm{keV} \mathrm{X}$-rays, using count rates of the order of $10^{3} \mathrm{c} /$ s. For X-ray spectrometry applications, fluorescence X-rays were induced in single and multi-

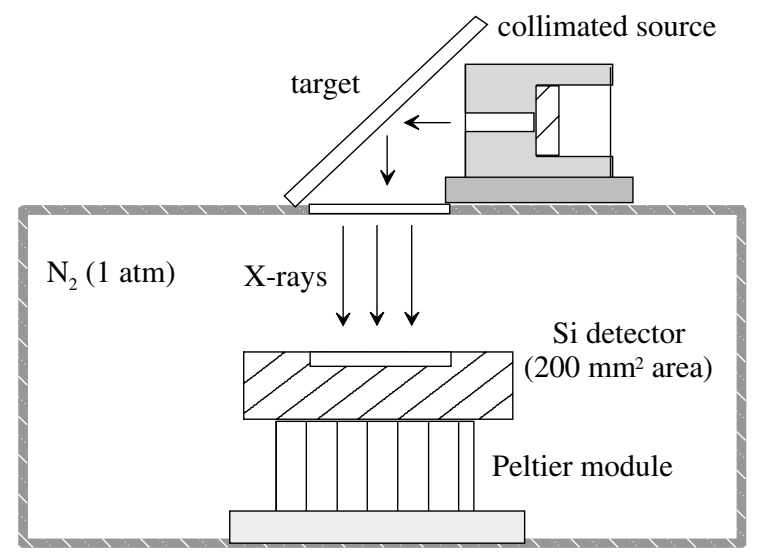

Fig. 1. Experimental setup for X-ray spectrometry applications.

element samples by ${ }^{55} \mathrm{Fe}$ and ${ }^{109} \mathrm{Cd}$ collimated sources, as shown in Fig. 1.

The LAAPD gain was calibrated with $170 \mathrm{~nm}$ UV light, chosen for being fully absorbed in the LAAPD before the multiplication region. The operating temperature was stabilised within $\pm 0.1{ }^{\circ} \mathrm{C}$.

In Fig. 2 we depict the LAAPD gain as a function of the operation temperature for different biasing voltages. For each voltage, the gain relative variation is almost constant through the measured temperature range, increasing from $2.4 \%$ to $4.5 \%$ per ${ }^{\circ} \mathrm{C}$, as the LAAPD biasing increases from 1633 to $1807 \mathrm{~V}$. This ratio is somewhat higher than that measured for visible light, which is only about $3 \%$ for the highest gains [10].

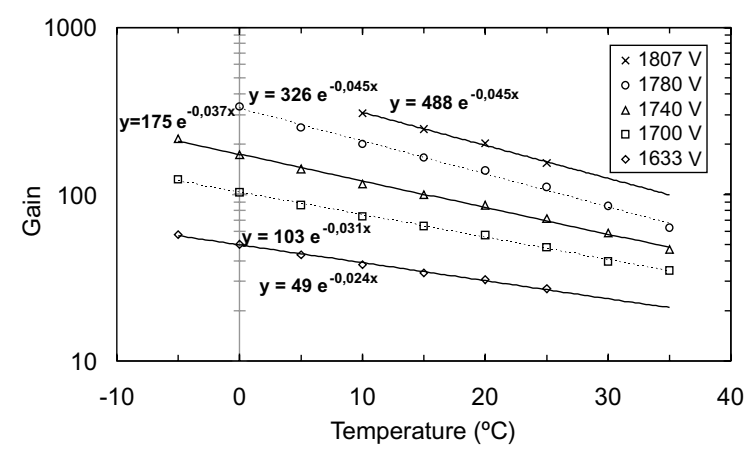

Fig. 2. LAAPD gain as a function of operating temperature for different bias voltages. 

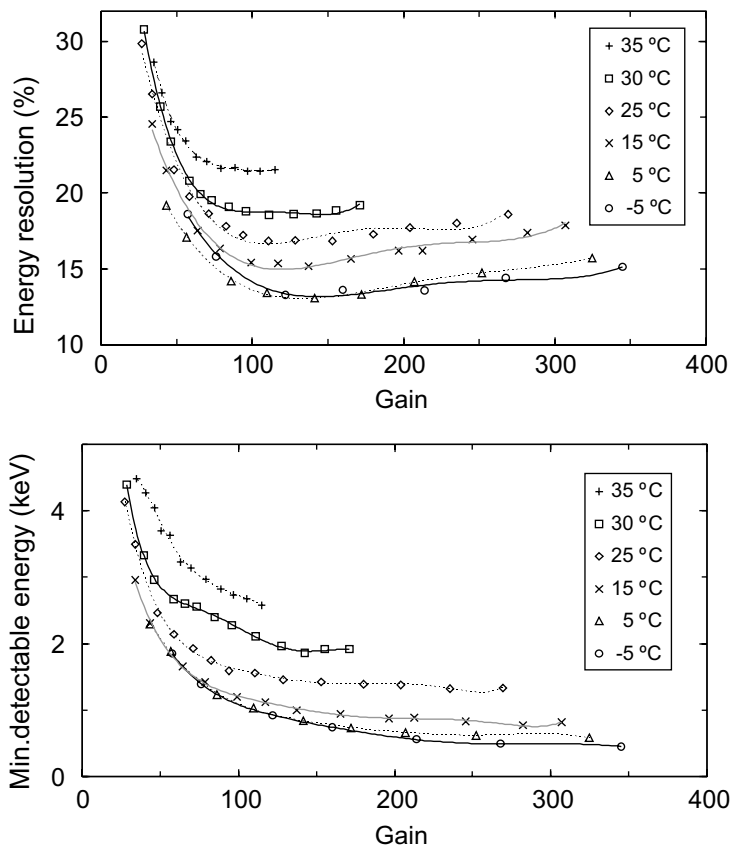

Fig. 3. LAAPD energy resolution and minimum detectable energy as a function of gain for different operating temperatures.

In Fig. 3 we present the detector energy resolution and the MDE as a function of the gain for different temperatures. The data clearly show the advantages of operating the photodiode at low temperatures. Best detector energy resolutions are achieved at gains of about 130, almost independent from the operation temperature, while the minimum detectable energy stabilises for gains above 200 .

Table 1 presents the LAAPD optimum operational characteristics for $5.9-\mathrm{keV} \mathrm{X}$-rays as well as the best MDE achieved, for the different studied temperatures. For temperatures below $5{ }^{\circ} \mathrm{C}$ both energy resolution and MDE do not improve significantly. At $5{ }^{\circ} \mathrm{C}$, the MDE is about a factor of 2 and 3 less than that achieved at 20 and $25^{\circ} \mathrm{C}$, respectively.

Fig. 3 reflects the effect of the dark current on the LAAPD performance. Higher dark current will result in worse device performance for both detector energy resolution and MDE. Detector energy resolution degradation with increasing detector biasing, for gains above $\sim 130$, cannot be related to the dark current only, since MDE stabilises. In fact, for gains above $\sim 130$ the contribution of the excess noise factor [10,11] becomes dominant. This factor is related to the gain fluctuations in the multiplication region and increases significantly for high gains [7].

The optimum operation gain depends on the individual LAAPD, and for each one a compromise between the gain and the device's dark current has to be established [8]. We note that, for low energy X-rays, this compromise is achieved for increasing gains since the noise contribution to the detector energy resolution becomes more significant as the X-ray energy approaches the MDE. For these cases, a higher gain will result in a lower MDE with a lower contribution of the noise to the energy resolution.

\section{X-ray spectrometry applications}

The energy linearity and energy resolution of the LAAPD were determined in the $2-20 \mathrm{keV}$ X-ray

Table 1

LAAPD optimum operational characteristics for $5.9-\mathrm{keV}$ X-rays and best MDE achieved for temperatures in the -5 to $35{ }^{\circ} \mathrm{C}$ range

\begin{tabular}{llllll}
\hline Temperature $\left({ }^{\circ} \mathrm{C}\right)$ & Voltage $(\mathrm{V})$ & Gain & En. Res. $(\%)$ & MDE $(\mathrm{keV})$ & Best MDE $(\mathrm{keV})$ \\
\hline-5 & 1700 & 122 & 13.3 & 0.9 & 0.5 \\
0 & 1720 & 131 & 13.1 & 0.9 & 0.5 \\
5 & 1740 & 141 & 13.1 & 0.8 & 0.6 \\
10 & 1747 & 126 & 14.6 & 1.0 & 0.7 \\
15 & 1760 & 127 & 15.2 & 1.1 & 0.8 \\
20 & 1770 & 124 & 15.7 & 1.2 & 1.0 \\
25 & 1794 & 130 & 16.8 & 1.5 & 1.3 \\
30 & 1820 & 128 & 18.5 & 2.0 & 2.6 \\
35 & 1847 & 115 & 21.5 & 2.6 & \\
\hline
\end{tabular}




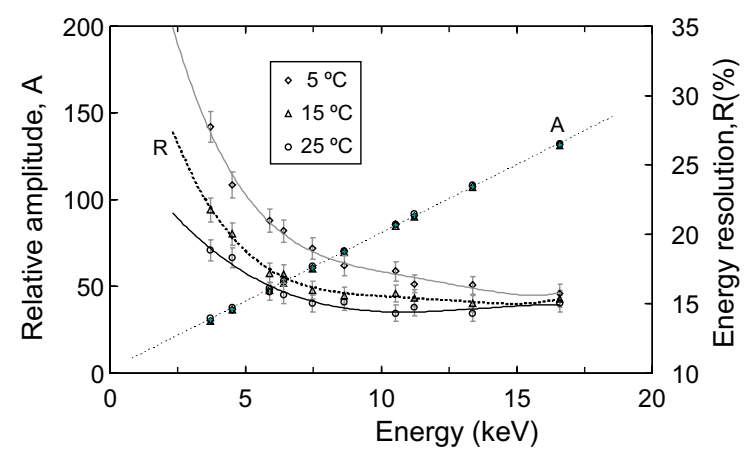

Fig. 4. Detector amplitude and energy resolution as a function of X-ray energy for different operation temperatures.

energy range using the fluorescence radiation induced in single-element samples. The centroid and relative full-width at half-maximum values of the pulse-height distributions are plotted in Fig. 4 as a function of the X-ray energy for different temperatures. At each temperature the LAAPD was operated with a biasing voltage corresponding to the gain of 130 .

While the detector energy linearity presents the same slope for all temperatures, the energy resolution improves with decreasing temperature. This is due to the decrease with temperature of the electronic noise which is more significant for lower energy values.

Fig. 5 depicts typical pulse-height distributions obtained for sulphur and biotite samples for different operation temperatures, excited with ${ }^{55} \mathrm{Fe}$ and ${ }^{109} \mathrm{Cd} \mathrm{X}$-ray sources. The spectra are similar to those obtained with a conventional PC.

Table 2 presents the LAAPD optimum operational characteristics for $2.3-\mathrm{keV}$ X-rays for each temperature. As discussed, best energy resolutions for 2.3-keV X-rays are achieved for higher gains than for 5.9-keV X-rays and best results are reached for lower operation temperatures. The MDE is about a factor of 2 higher than achieved for the conditions at which $5.9-\mathrm{keV} \mathrm{X}$-ray peaks were obtained due to the much lower count rate reached for the sulphur peak, $4 \mathrm{c} / \mathrm{s}$.

\section{Conclusions}

Detector energy resolution and lowest detectable X-ray energy present a strong improvement as
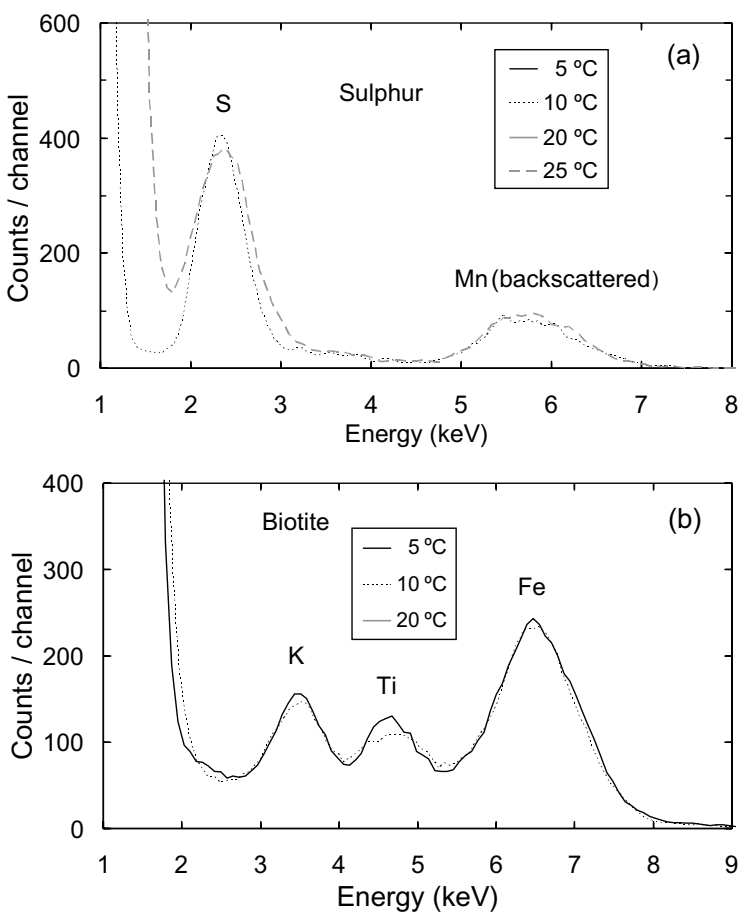

Fig. 5. Pulse-height distributions obtained with the Peltiercooled LAAPD for sulphur (a) and biotite (b) fluorescence Xrays and for different operation temperatures.

Table 2

LAAPD optimum operational characteristics for $2.3-\mathrm{keV}$ Xrays achieved for temperatures in the -5 to $25^{\circ} \mathrm{C}$ range

\begin{tabular}{lllll}
\hline $\begin{array}{l}\text { Tempera- } \\
\text { ture }\left({ }^{\circ} \mathrm{C}\right)\end{array}$ & $\begin{array}{l}\text { Volt- } \\
\text { age } \\
(\mathrm{V})\end{array}$ & Gain & $\begin{array}{l}\text { En. } \\
\text { Res. } \\
(\%)\end{array}$ & $\begin{array}{l}\text { MDE } \\
(\mathrm{keV})\end{array}$ \\
\hline-5 & 1766 & 345 & 22.4 & 1.1 \\
0 & 1780 & 335 & 22.8 & 1.2 \\
5 & 1793 & 325 & 23.3 & 1.1 \\
10 & 1807 & 307 & 23.8 & 1.3 \\
15 & 1815 & 282 & 24.1 & 1.3 \\
20 & 1820 & 247 & 27.4 & 1.4 \\
25 & 1838 & 235 & 29.9 & 1.7 \\
\hline
\end{tabular}

the operation temperature is reduced from 25 to $10{ }^{\circ} \mathrm{C}$ and much slower improvements for temperatures below $10^{\circ} \mathrm{C}$. While for $5.9-\mathrm{keV}$ X-rays the performance does not improve significantly for temperatures below $5-10{ }^{\circ} \mathrm{C}$, for lower energies lower operation temperatures may further improve the detector performance. Additionally, while for 5.9-keV X-rays optimum gains, around 130, are 
only slightly dependent on the operation temperature, for low energy X-rays optimum performance is obtained for increasing gains, reaching values above 300 for operation temperatures below $10{ }^{\circ} \mathrm{C}$ and for $2.3-\mathrm{keV}$ X-rays.

\section{References}

[1] A.Q.R. Baron, S.L. Ruby, Nucl. Instr. and Meth. A 343 (1994) 517.

[2] E.M. Gullikson, E. Gramsch, M. Szawlowski, Appl. Opt. 34 (1995) 4662.

[3] J.P. Pansart, Nucl. Instr. and Meth. A 387 (1997) 186.
[4] K. Deiters, Y. Musienko, S. Nicol, et al., Nucl. Instr. and Meth. A 442 (2000) 193.

[5] R. Farrel, F. Olschner, K. Shah, M.R. Squillante, Nucl. Instr. and Meth. A 387 (1997) 194.

[6] A. Ruru Chen, A. Fremout, S. Tavernier, et al., Nucl. Instr. and Meth. A 433 (1999) 637.

[7] M. Moszynski, M. Szawlowski, M. Kapusta, et al., Nucl. Instr. and Meth. A 442 (2000) 230.

[8] L.M.P. Fernandes, J.A.M. Lopes, J.M.F. dos Santos, C.A.N. Conde, X-ray Spectrom. 30 (2001) 164.

[9] Advanced Photonix Inc., 1240 Avenida Acaso, Camarillo, CA 93012, USA.

[10] Advanced Photonix Inc., Windowless large area APDs ( $5 \times 0$ deep-UV series), Application Notes.

[11] J.A.M. Lopes, J.M.F. dos Santos, C.A.N. Conde, IEEE Trans. Nucl. Sci. 48 (2001) 312. 